\title{
Cathepsin B and Cystatin A as Indicators of a Separate Apoptotic Pathway in HIV-I Infection Pål Voltersvik ${ }^{1}$, Leif Bostad ${ }^{2}$, AnneMa Dyrhol-Riise ${ }^{1,3}$ and Birgitta Åsjö*ł1
}

\author{
Address: ${ }^{1}$ Center f. Res. in Virology, The Gade Institute, Univ. of Bergen, Bergen, Norway, ${ }^{2}$ Dept. of Pathology, Bergen, Norway and ${ }^{3}$ Inst. of \\ Medicine Haukeland University Hospital, Bergen, Norway \\ Email: Birgitta Åsjö* - birgitta.asjo@gades.uib.no \\ * Corresponding author ‡Presenting author
}

from 2005 International Meeting of The Institute of Human Virology Baltimore, USA, 29 August - 2 September 2005

Published: 8 December 2005

Retrovirology 2005, 2(Suppl I):PII doi:I0.I |86/I742-4690-2-SI-PI I

Apoptosis has been proposed to explain the dysfunction in HIV-1 infection and FAS has been given a pivotal role. However, apoptosis in lymphoid follicles has also been explained by a follicular dendritic cell (FDC) dependent pathway regulated by a cathepsin-dependent endonuclease activity in germinal centre (GC) cells. Cystatin A is present in FDCs and is a natural inhibitor of cysteine proteinase, as Cathepsin B. As yet, the Cystatin A and Cathepsin $B$ interaction in HIV-1 infection has not been studied.

\section{Methods}

Tonsillar tissue was obtained from 20 patients at various stages of HIV-1 infection and 10 controls. Eleven of the patients received HAART for 48 weeks. Cathepsin B, Cystatin A, FAS(CD95) and HIV-1 p24 in the GC cells were analyzed by immunohistochemi-cal staining. Cathepsin B/Cystatin A ratios were calculated for controls and for patients before and after 48 weeks of therapy.

\section{Results}

Cathepsin B/Cystatin A ratio was 2-fold higher in patients as compared to controls; 1.03 and 0.43 , respectively. After 48 weeks of therapy, this ratio was normalized (0.32). In patients, Cathepsin B correlated negatively with Cystatin A $(r=-0.686, p=0.002)$, and both markers correlated with the $\mathrm{p} 24$ antigen; $r=0.777(\mathrm{p}=0.001)$ and $\mathrm{r}=-0.622$ $(p=0.013)$, respectively. In multiple regression analysis presence of p24 antigen could not fully explain this relationship. There was no correlation with FAS(CD 95) for these parameters.

\section{Conclusion}

A 2-fold higher Cathepsin B/Cystatin A ratio was found in patients before HAART, suggesting a HIV-1 driven cathepsin-dependent pathway of apoptosis. Thus, Cathepsin B and Cystatin A possibly represent an apoptotic pathway distinguishable from the FAS-FAS Ligand pathway. 